\title{
The relationship between thyroid autoantibody positivity and abnormal pregnancy outcomes and miscarriage in euthyroid patients
}

\author{
Selçuk Kaplan
}

Department of Gynaecology and Obstetrics, School of Medicine, Adıyaman University, Adıyaman, Turkey

\begin{abstract}
Introduction: The aim of this study is to determine the relationship between autoantibodies against TPO (TPOAb) and thyroid receptor antibody (TRAb) autoantibody positivity and abnormal pregnancy outcomes and miscarriage in euthyroid pregnant women.

Material and methods: This study is a retrospective case-control study conducted by examining the data of 16,876 women who applied to Adiyaman Training and Research Hospital for regular obstetric examination between December 2017 and March 2020. Analyses were performed to compare the risks of gestational diabetes mellitus (GDM), preeclampsia, placenta previa, placenta abruption, foetal growth restriction (FGR), foetal distress, stillbirth, preterm birth, and miscarriage. $P<0.05$ was considered statistically significant.

Results: In the group with TPO +/TRAb-, placenta previa risk (odds ratio $(\mathrm{OR})=2.26,1.61-3.17, p<0.001$ ), placenta abruption risk $(\mathrm{OR}=4.24,2.14-8.41, p<0.001)$, FGR risk $(\mathrm{OR}=1.28,1.11-1.48, p<0.001)$, and miscarriage risk $(\mathrm{OR}=1.63,1.38-1.92, p<0.001)$ increased. In the group with TPO-/TRAb+, risk of preeclampsia $(\mathrm{OR}=2.58,2.08-3.20, p<0.001)$, risk of placenta previa $(\mathrm{OR}=2.40,1.51-3.80, p<0.001)$, and risk of miscarriage $(\mathrm{OR}=1.29,1.01-1.66, p=0.004)$ increased. In the group with TPO+/TRAb+, GDM risk $(\mathrm{OR}=1.86,1.44-2.41, p<0.001)$, placenta previa risk $(\mathrm{OR}=4.76,3.30-6.86, p<0.001)$, and miscarriage risk $(\mathrm{OR}=1.67,1.31-2.11, p<0.001)$ increased.

Conclusions: Thyroid autoantibody positivity is associated with negative perinatal outcomes such as miscarriage, placenta previa, GDM, and preeclampsia, regardless of thyroid hormone levels. Thyroid autoantibody detection in early visits of pregnant women should be a warning for the clinician.

Key words: diabetes, gestational, abortion, spontaneous, foetal membranes, preterm delivery, preeclampsia, pregnancy complications, thyroiditis, autoimmune, TPO protein, human, thyroid stimulation-blocking antibody.
\end{abstract} cine, Adıyaman University, Atatürk Blv. No: 411, 02200 Merkez, Adıyaman, phone: +90 41622338 00, fax: +90 41622338 36, e-mail: kaplan_2384@hotmail.com 


\section{Introduction}

Approximately $10 \%$ of the general population and at least $2-3 \%$ of pregnant women suffer from autoimmune thyroid diseases (ATD) [1]. Thyroid receptor antibody (TRAb) is an important autoantibody detected in patients with autoimmune thyroid disease [2]. Thyroid peroxidase (TPO) is a key enzyme involved in thyroid hormone synthesis [3]. The presence of autoantibodies against TPO (TPOAb) and the secondary developing response are considered as indicators of thyroid autoinflammation and can be detected in 10-20\% of women in the reproductive period $[4,5]$. It is widely known that negative perinatal outcomes are associated with thyroid dysfunction in pregnancy [6]. Moreover, thyroid autoimmunity appears to be associated with some pregnancy complications, including miscarriage and preterm birth [7]. In addition, women with recurrent foetal loss, infertility, or miscarriage are more likely to have thyroid autoantibodies than women without thyroid autoantibodies positivity [8]. It has been shown that the presence of thyroid autoantibodies causes changes in the immune system at the foetal maternal interface, causing miscarriage, preterm labour, and adverse advanced pregnancy results [9]. Therefore, it is important to raise awareness of autoimmune thyroid diseases and the prevention of complications related to their treatment during pregnancy.

The aim of this study is to determine the relationship between TPOAb and TRAb autoantibody positivity and abnormal pregnancy outcomes such as gestational diabetes mellitus, preeclampsia, abnormal placental localisation, and miscarriage in euthyroid pregnant women.

\section{Material and methods}

This study is a retrospective case-control study conducted by examining the data of 16,876 women who applied to Adıyaman Training and Research Hospital for regular ob- stetric examination between December 2017 and March 2020. Local Ethics Committee approval was received for the study. This study was conducted in accordance with the principles of the Helsinki Declaration.

Patients with clinical symptoms of hypothyroidism and hyperthyroidism [10], whose thyroid hormone levels were not within normal limits, had a history of antithyroid drug therapy or a history of thyroid hormone replacement, patients with non-thyroid autoimmune disease clinics (rheuomatoid arthritis, systemic lupus erythematosus etc.), and patients with immunosuppressive therapy in the preceding 6 months, were excluded from the study. The criteria for inclusion in the study were to have performed all controls in our centre during pregnancy, to have determined the values of thyroid autoantibodies (TPOAb and TRAb) at the first visit, and to be in the 18-40 years age range. One thousand eight hundred and thirty-one patients with a diagnosis of hypothyroidism, 463 patients with a diagnosis of hyperthyroidism, 934 patients with a diagnosis of subclinical hypothyroidism, and 217 patients with a diagnosis of subclinical hyperthyroidism were excluded from the study.

TSH and FT4 concentrations were within normal ranges in all selected women [11]. Specifically, thyroid stimulating hormone (TSH) and free T4 hormone (FT4) values were $0.09-3.47 \mathrm{mIU} / \mathrm{l}$ and $6.00-12.25 \mathrm{ng} / \mathrm{l}$ in early pregnancy, respectively; $0.20-3.81 \mathrm{mIU} / \mathrm{l}$ and $4.30-9.74 \mathrm{ng} / \mathrm{l}$ in middle pregnancy; and $0.67-4.99 \mathrm{mIU} / \mathrm{l}$ and $4.56-8.50 \mathrm{ng} / \mathrm{l}$ in late pregnancy. TPOAb > C50 IU/ml and TRAb > $1.75 \mathrm{IU} / \mathrm{l}$ were considered positive.

As a result, 13,431 euthyroid [12] patients were included in the study. The patients were divided into four groups according to serum TPOAb and TRAb results. TPOAb-/ TRAb- (83.4\%, 11,204/13,431), TPOAb+/TRAb- (8.8\%, $1181 / 13,431)$, TPOAb-/TRAb+ $(4.1 \%, 544 / 13,431)$, and $\mathrm{TPOAb}+/ \mathrm{TRAb}+(3.7 \%, 502 / 13,431)$ (Figure 1). The TPOAb-/TRAb- group was determined as the control group.

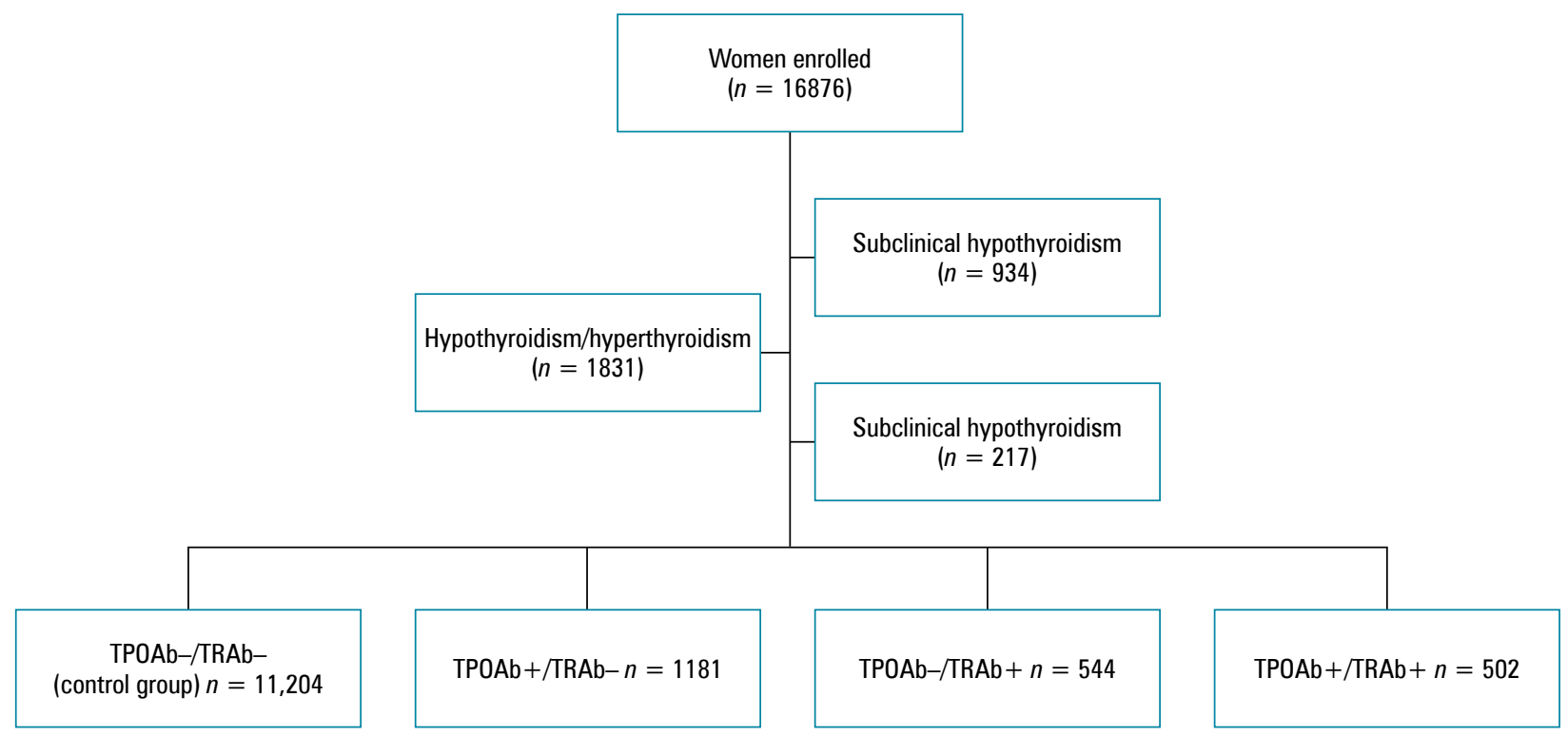

Figure 1. Flowchart of study population

$n$ - number of patients, TPOAb - thyroid peroxidase autoantibody, TRAb - thyroid receptor autoantibody. 


\section{Abnormal pregnancy outcome assessment}

We searched for nine abnormal pregnancy results defined as below. Gestational diabetes mellitus (GDM): oral glucose tolerance test (OGTT) was performed using $75 \mathrm{~g}$ of oral glucose solution; fasting plasma glucose $92 \mathrm{mg} / \mathrm{dl}(5.1$ $\mathrm{mmol} / \mathrm{l}), 1$-hour plasma glucose $180 \mathrm{mg} / \mathrm{dl}(10 \mathrm{mmol} / \mathrm{l})$, 2-hour plasma glucose $153 \mathrm{mg} / \mathrm{dl}$ ( $8.5 \mathrm{mmol} / \mathrm{l})$ [12]. Preeclampsia: systolic blood pressure $140 \mathrm{~mm} \mathrm{Hg}$ or diastolic blood pressure $\geq 90 \mathrm{~mm} \mathrm{Hg}$ after 20 weeks in a pregnant woman without a history of hypertension and the coexistence of one or more of the following new onset conditions: proteinuria, renal insufficiency (creatinine $>90 \mu \mathrm{mol} / \mathrm{l}$ ), liver involvement (elevated transaminases and/or severe right upper quadrant or epigastric pain), neurological complications (headaches, altered mental status; examples include eclampsia, stroke, blindness, hyperreflexia or clonus, when accompanied by hyperreflexia), and haematological complications (thrombocytopaenia, haemolysis, disseminated intravascular coagulation). Placenta previa: the placenta advances to the lower uterine segment, partially or completely covering the entrance to the cervix. Abruptio placentae: the placental membrane is partially or completely separated from the uterus endometrium before birth. Foetal growth restriction (FGR): a newborn who is small for gestational date is one whose weight falls below the $10^{\text {th }}$ percentile. Faetal distress: worsening of the foetus in antepartum or intrapartum period due to foetal hypoxia. Stillbirth: foetal death after 20 weeks of pregnancy. Preterm delivery: a baby born between 28 and 37 weeks of age. Pregnancy loss: abortion before $20^{\text {th }}$ gestational week.

\section{Statistical analysis}

Statistical analyses were performed using SPSS 22 (International Business Machines Corp., Armonk, New York). Qualitative data were expressed as frequency, percentage, median (minimum-maximum), and mean \pm standard deviation. Pearson's $\chi^{2}$ test was used to determine the significance of the difference between the two percentages, and the paired $t$-test was used for binary dependent groups fitting the normal distribution. Pearson's $\chi^{2}$ test was used to calculate the differences of the groups for GDM, preeclampsia, placenta previa, placenta abruption, FGR, foetal distress, stillbirth, preterm delivery, and miscarriage, compared to the control group.

Using univariate conditional logistic regression analysis, we calculated the odds ratio (OR) and $95 \%$ confidence intervals (CI) for each variable. A multivariate logistic regression analysis was used to adjust potential contradictions and calculate the corrected adjusted odds ratio (AOR). $P \leq 0.050$ was considered statistically significant.

\section{Results}

The incidence of adverse pregnancy outcomes by groups is given in Table I.

There is no statistically significant difference between the groups for the risk of preterm delivery, foetal distress, or stillbirth $(p>0.05)$ (Table I).

In the group with $\mathrm{TPO}+/ \mathrm{TRAb}-$, placenta previa risk $(\mathrm{OR}=2.26,1.61-3.17, p<0.001)$, placenta abruption risk $(\mathrm{OR}=4.24,2.14-8.41, p<0.001)$, FGR risk $(\mathrm{OR}=1.28$, $1.11-1.48, p<0.001)$, and abortion risk $(\mathrm{OR}=1.63,1.38-$ $1.92, p<0.001)$ increased (Table II).

In the group with TPO-/TRAb+, risk of preeclampsia $(\mathrm{OR}=2.58,2.08-3.20, p<0.001)$, risk of placenta previa $(\mathrm{OR}=2.40,1.51-3.80, p<0.001)$, and risk of abortion $(\mathrm{OR}=1.29,1.01-1.66, p=0.004)$ increased (Table II).

In the group with $\mathrm{TPO}+/ \mathrm{TRAb}+, \mathrm{GDM}$ risk $(\mathrm{OR}=1.86$, $1.44-2.41, p<0.001)$, placenta previa risk $(\mathrm{OR}=4.76,3.30-$ $6.86, p<0.001)$, and abortion risk $(\mathrm{OR}=1.67,1.31-2.11$, $p<0.001)$ increased (Table II).

\section{Discussion}

According to the results of this study, TPOAb and TRAb positivity increase the risk of placenta previa and miscarriage. TPOAb positivity increases the risk of FGR, TRAb

Table I. Incidences of adverse pregnancy outcomes by groups

\begin{tabular}{|l|c|c|c|c|}
\hline Parameter & TPOAb-/TRab- & TPOAb + TRAb- & TPOAb-/TRAb & TPOAb $+/ T R A b+$ \\
\hline Number of subjects & 11204 & 1181 & 544 & 502 \\
\hline GDM & $933(8.3 \%)$ & $82(7 \%)$ & $57(10.5 \%)$ & $73(14.6 \%)$ \\
\hline Preeclampsia & $1074(9.6 \%)$ & $109(9.2 \%)$ & $117(21.5 \%)$ & $44(8.7 \%)$ \\
\hline P. previa & $184(1.7 \%)$ & $43(3.7 \%)$ & $21(3.9 \%)$ & $37(7.4 \%)$ \\
\hline A. placenta & $27(0.3 \%)$ & $12(1.01 \%)$ & - & - \\
\hline FGR & $2127(18.9 \%)$ & $274(23.2 \%)$ & $41(7.5 \%)$ & $49(9.8 \%)$ \\
\hline Foetal distress & $311(2.8 \%)$ & $44(3.7 \%)$ & $19(3.5 \%)$ & $17(3.4 \%)$ \\
\hline Stillbirth & $11(0.1 \%)$ & $3(0.3 \%)$ & - & - \\
\hline Preterm delivery & $1819(16.2 \%)$ & $153(13 \%)$ & $64(11.8 \%)$ & $76(15.1 \%)$ \\
\hline Miscarriage & $1264(11.3 \%)$ & $203(17.2 \%)$ & $77(14.2 \%)$ & $88(17.5 \%)$ \\
\hline
\end{tabular}

TPOAb - thyroid peroxidase autoantibody, TRAb - thyroid receptor autoantibody, GDM - gestational diabetes mellitus, FGR - foetal growth restriction, P - placenta, A - abruptio. 
Table II. Negative perinatal results by groups and multivariate logistic regression analysis results for miscarriage

\begin{tabular}{|c|c|c|c|c|c|c|}
\hline Parameter & $\mathrm{TPOAb}+/ \mathrm{TRAb}-\mathrm{OR}$ & $P$-value & $\mathrm{TPOAb}-/ \mathrm{TRAb}+\mathrm{OR}_{2}$ & $P$-value ${ }_{1}$ & $\mathrm{TPOAb}+/ \mathrm{TRAb}+\mathrm{OR}_{3}$ & $P$-value ${ }_{3}$ \\
\hline GDM & $0.81(0.64-1.03)$ & 0.091 & $1.28(0.96-1.70)$ & 0.085 & OR: $1.86(1.44-2.41)$ & $<0.001$ \\
\hline Preeclampsia & $0.95(0.78-1.17)$ & 0.69 & $2.58(2.08-3.20)$ & $<0.001$ & OR: $0.90(0.66-1.24)$ & 0.540 \\
\hline P. previa & $2.26(1.61-3.17)$ & $<0.001$ & $2.40(1.51-3.80)$ & $<0.001$ & OR: $4.76(3.30-6.86)$ & $<0.001$ \\
\hline A. placenta & $4.24(2.14-8.41)$ & $<0.001$ & - & - & - & - \\
\hline IUGR & $1.28(1.11-1.48)$ & $<0.001$ & $0.34(0.25-0.48)$ & $<0.001$ & OR: $0.46(0.34-0.62)$ & $<0.001$ \\
\hline Foetal distress & $1.35(0.98-1.87)$ & 0.064 & $1.27(0.79-2.03)$ & 0.32 & OR: $1.22(0.74-2.01)$ & 0.410 \\
\hline Stillbirth & $2.59(0.72-9.30)$ & 0.14 & - & - & - & - \\
\hline Preterm delivery & $0.76(0.64-0.91)$ & 0.003 & $0.68(0.52-0.89)$ & 0.006 & OR: 0.92 (0.71-1.18) & 0.510 \\
\hline Miscarriage & $1.63(1.38-1.92)$ & $<0.001$ & $1.29(1.01-1.66)$ & 0.004 & OR: $1.67(1.31-2.11)$ & $<0.001$ \\
\hline
\end{tabular}

OR - odds ratio, TPOAb - thyroid peroxidase autoantibody, TRAb - thyroid receptor autoantibody, GDM - gestational diabetes mellitus, IUGR - intrauterine growth restriction, $\mathrm{P}$ - placenta, A - abruptio.

positivity preeclampsia, and TPO and TRAb positivity increases the risk of GDM.

In a prospective cohort study by Liu et al. including subclinical hypothyroid pregnancies and euthyroid pregnancies with TPO antibody positivity, the risk of miscarriage increased in euthyroid patients with TPO antibody positivity [13]. In addition, another study reported that TPO positivity was associated with miscarriage [14]. However, in another study, although the rate of miscarriage was high in patients with TPOAb elevation, there was no statistically significant difference [15]. Li et al. in a recent study found that TRab positivity were significantly higher in women with pregnancy loss [16]. In this study, the risk of miscarriage in the TPOAb+ and TRAb+, and TPOAb and TRAb+ patient groups was reported to be increased. Thyroid autoantibody positivity increases the risk of miscarriage regardless of thyroid hormone levels. Therefore, the determination of TPOAb and TRAb positivity in pregnant women becomes important. However, there is no evidence showing the effectiveness of treatment in pregnant women [17].

Thyroid autoantibody positivity leads to the activation of a general immune response against the foetal-maternal interface of the immune system and leads to preterm delivery [14]. In a recent study, it was reported that the risk of preterm delivery increased with TPOAb positivity [18]. In addition, there are other studies showing that the risk of preterm delivery increases with TPO positivity [9, 19]. Consistent with the previous study, the results of this study also increased the risk of preterm.

However, in a prospective study, there was no relationship between TPOAb and TgAb positivity and preterm delivery risk [20]. Moreover, there is a current study showing that there is no relationship between TGAb positivity and preterm delivery [16]. In this study, there was no relationship between TPOAb positivity or TRAb positivity and preterm delivery risk. This contradiction with the mentioned studies $[9,18,19]$ may be related to the selection of our patient groups among euthyroid patients [20].

In a study conducted in patients with subclinical thyroid dysfunction, there was no relationship between the risk of preeclampsia and positivity of thyroid autoantibodies [21]. However, in a study conducted in Iran, TPOAb positivity increased the risk of preeclampsia [22]. In a study investigating the relationship between antibodies showing receptor activation and preeclampsia, it was stated that TRAbs may cause TSH-like activation and may be involved in G protein-coupled receptor (GPCR) activation, and can bind to Angiotensin I receptor that plays a role in the pathophysiology of preeclampsia [23]. According to this study, there was no relationship between TPOAb positivity and preeclampsia, but the risk of gestational hypertension increased with TRAb positivity. There is no strong evidence to show the relationship between TPOAb positivity and hypertensive diseases [17].

In a study conducted in a group of patients with high TSH levels and TPOAb positivity, it was reported that TPOAb positivity increased the risk of GDM [24].

In addition, it has been reported that TPOAb prevalence is high in GDM patients [25]. However, in a recent cohort study there was no relationship between TPOAb positivity and GDM [26]. It was reported in another study that women with GDM had higher prevalence of TPOAb and TGAb, and it was reported that autoimmune thyroid diseases can be triggered by hyperglycaemia [27]. Moreover, another study stated that hypothyroidism is a risk factor for GDM, but autoantibody positivity is not associated with GDM risk [28]. In this study, the risk of GDM was increased in pregnant women with TPOAb and TRAb positivity. There is a significant but weak relationship between GDM and thyroid autoantibody positivity, and thyroid autoantibody positivity may not be a risk factor for GDM in euthyroid patients. These data should be considered in the follow-up of patients with thyroid autoantibody positivity [29].

Adverse results such as abnormalities in lipid metabolism, coagulation disorders, impairment in endothelial functions, and arterial intimal thickening have been reported in patients with positive thyroid autoantibody. These adverse developments in the placental vascular bed may increase the risk of abruptio placenta [30]. There are some studies showing that a relationship between TPOAb 
positivity and placenta abruptio [31, 32]. Similarly, in this study, the risk of placenta abruption was increased in pregnant women with TPOAb positivity. In a study investigating the positivity of TPOAb and TgAb with perinatal outcomes in euthyroid patients, there was no relationship between placenta previa and autoantibody positivity [33]. In addition, in a meta-analysis investigating the perinatal results of patients with subclinical hypothyroidism, it was stated that there was no relationship between hypothyroidism and placenta previa [34]. In this study, the risk of placenta previa was found to be increased in pregnant women with TPOAb and TRAb positivity.

This study has some limitations. First of all, this study is a retrospective study, so it can be biased. However, we tried to eliminate this by obtaining the data from recorded and written results. Secondly, this is a single-centre study, and the number of patients in the case group is low compared to the control group population. These limitations should be considered before generalising the results of the study to the society. Large, multicentre, and prospective studies may be needed.

\section{Conclusions}

Thyroid autoantibody positivity is associated with low perinatal outcomes such as low levels of thyroid hormones and negative perinatal outcomes such as placenta previa and miscarriage. Thyroid autoantibody positivity in early visits of pregnant women should be a warning for the clinician. The adverse effects of thyroid autoantibody positivity should not be ignored, and the clinician should determine thyroid autoantibody values in early pregnancy visits and take precautions against adverse pregnancy outcomes.

\section{Conflict of interest}

The authors declare no conflict of interest.

\section{References}

1. Matthews DC, Syed AA. The role of TSH receptor antibodies in the management of Graves' disease, Eur J Intern Med 2011; 22: 213-6.

2. Green AS, Roman SH, Cobin RHE, eI-Harazy E, Marfang MA, Davies TF. Detection of at-risk pregnancy by means of highly sensitive assays for thyroid autoantibodies. JAMA 1990; 264: 1422-5.

3. Ruf J, Carayon P. Structural and functional aspects of thyroid peroxidase. Arch Biochem Biophys 2006; 445: 269-77.

4. Prummel MF, Wiersinga WM. Thyroid peroxidase autoantibodies in euthyroid subjects. Best Pract Res Clin Endocrinol Metab 2005; 19: 1-15.

5. Balucan FS, Morshed SA, Davies TF. Thyroid autoantibodies in pregnancy: their role, regulation and clinical relevance. J Thyroid Res 2013; 2013: 182472 .

6. Lumen A, George NI. Estimation of iodine nutrition and thyroid function status in late-gestation pregnant women in the United States: development and application of a population-based pregnancy model. Toxicol Appl Pharmacol 2017; 314: 24-38.

7. Negro R, Schwartz A, Gismondi R, Tinelli A, Mangieri T, Green AS. Thyroid antibody positivity in the first trimester of pregnancy is associated with negative pregnancy outcomes. J Clin Endocrinol Metab 2011; 96: e920-4.

8. Negro R, Schwartz A, Green AS. Impact of levothyroxine in miscarriage and preterm delivery rates in first trimester thyroid antibody-positive women with TSH less than $2.5 \mathrm{mIU} / \mathrm{L}$. J Clin Endocrinol Metab 2016; 101: 3685-90.

9. Thangaratinam S, Tan A, Knox E, Kilby MD, Franklyn J, Coomarasamy A. Association between thyroid autoantibodies and miscarriage and preterm birth: meta-analysis of evidence. BMJ 2011; 342: d2616.

10. Garber JR, Cobin RH, Gharib H, et al. American Association of Clinical Endocrinologists and American Thyroid Association Taskforce on Hypothyroidism in Adults, Clinical practice guidelines for hypothyroidism in adults: cosponsored by the American Association of Clinical Endocrinologists and the American Thyroid Association. Thyroid 2012; 22: 1200-35.

11. Lu XM, Chen LM, Yang H, et al. Trimester specific reference data and variation of thyroid hormones for normal pregnancy. J Med Res 2012; 41: 70-3.

12. Coustan DR, Lowe LP, Metzger BE, Dyer AR; International Association of Diabetes and Pregnancy Study Groups. The Hyperglycemia and Adverse Pregnancy Outcome (HAPO) study: paving the way for new diagnostic criteria for gestational diabetes mellitus. Am J Obstet Gynecol 2010; 202: 654.e1-6.

13. Liu H, Shan Z, Li C, et al. Maternal subclinical hypothyroidism, thyroid autoimmunity, and the risk of miscarriage: a prospective cohort study. Thyroid 2014; 24: 1642-9.

14. Rajput R, Yadav T, Seth S, Nanda S. Prevalence of thyroid peroxidase antibody and pregnancy outcome in euthyroid autoimmune positive pregnant women from a Tertiary Care Center in Haryana. Indian J Endocrinol Metab 2017; 21: 577-80.

15. Bhattacharyya R, Mukherjee K, Das A, Biswas MR, Basunia SR, Mukherjee A. Anti-thyroid peroxidase antibody positivity during early pregnancy is associated with pregnancy complications and maternal morbidity in later life. J Nat Sci Biol Med 2015; 6: 402-5.

16. Li Y, Xu T, Mo Q, Fu W, Yao C. Thyrotropin receptor antibody: a novel risk indicator for pregnancy loss. Clin Biochem 2019; 64: 44-8.

17. Dhillon-Smith RK, Coomarasamy A. TPO antibody positivity and adverse pregnancy outcomes. Best Practice Res Clin Endocr Metabol 2020; 34: 101433

18. Consortium on Thyroid and Pregnancy - Study Group on Preterm Birth; Korevaar TIM, Derakhshan A, Taylor PN, et al. Association of thyroid function test abnormalities and thyroid autoimmunity with preterm birth: a systematic review and meta-analysis. JAMA 2019; 322: 632-41.

19. He X, Wang P, Wang Z, He X, Xu D, Wang B. Thyroid antibodies and risk of preterm delivery: a meta-analysis of prospective cohort studies. Eur J Endocrinol 2012; 167: 455-64.

20. Ashoor G, Maiz N, Rotas M, Jawdat F, Nicolaides KH. Maternal thyroid function at 11 to 13 weeks of gestation and subsequent fetal death. Thyroid 2010; 20: 989-93.

21. van den Boogaard E, Vissenberg R, Land JA, et al. Significance of (sub)clinical thyroid dysfunction and thyroid autoimmunity before conception and in early pregnancy: a systematic review. Hum Reprod Update 2011; 17: 605-19.

22. Saki F, Dabbaghmanesh MH, Ghaemi SZ, Forouhari S, Omrani GR, Bakhshayeshkaram M. Thyroid autoimmunity in pregnancy 
and its influences on maternal and fetal outcome in Iran (a prospective study). Endocr Res 2015; 40: 139-45.

23. Yang X, Kellems RE. Receptor-activating autoantibodies and disease: preeclampsia and beyond. Exp Rev Clin Immunol 2011; 7: 659-74.

24. Karakosta P, Alegakis D, Georgiou V, et al.. Thyroid dysfunction and autoantibodies in early pregnancy are associated with increased risk of gestational diabetes and adverse birth outcomes. J Clin Endocrinol Metab 2012; 97: 4464-72.

25. Bitterman O, Bongiovanni M, Giuliani C, Roma G, Toscano V, Napoli A. Anti thyroperoxidase and anti thyroglobulin antibodies in diabetic pregnancies. J Endocrinol Invest 2014; 37: 911-5.

26. Plowden TC, Schisterman EF, Sjaarda LA, et al. Subclinical hypothyroidism and thyroid autoimmunity are not associated with fecundity, pregnancy loss, or live birth. J Clin Endocrinol Metab 2016; 101: 2358-65.

27. Vitacolonna E, Lapolla A, Nenno BD, et al. Gestational diabetes and thyroid autoimmunity. Int J Endocrinol 2012; 2012: 867415.

28. Mannisto T, Vaarasmaki M, Pouta A. Thyroid dysfunction and autoantibodies during pregnancy as predictive factors of pregnancy complications and maternal morbidity in later life. J Clin Endocrinol Metab 2010; 95: 1084-94.

29. Yang Y, Li Q, Wang Q, Ma X. Thyroid antibodies and gestational diabetes mellitus: a meta-analysis. Fertil Steril 2015; 104: 665-71.e3.

30. Cellini M, Santaguida MG, Stramazzo I, et al. Recurrent pregnancy loss in women with Hashimoto's thyroiditis with concurrent non-endocrine autoimmune disorders. Thyroid 2020; 30: 457-62.

31. Abbassi-Ghanavati M, Casey BM, Spong CY, McIntire DD, Halvorson LM, Cunningham FG. Pregnancy outcomes in women with thyroid peroxidase antibodies. Obstet Gynecol 2010; 116: 381-6.

32. Haddow JE, McClain MR, Palomaki GE, et al. Thyroperoxidase and thyroglobulin antibodies in early pregnancy and placental abruption. Obstet Gynecol 2011; 117: 287-92.

33. Chen LM, Zhang Q, Si GX, et al. Associations between thyroid autoantibody status and abnormal pregnancy outcomes in euthyroid women. Endocrine 2015; 48: 924-8.

34. Maraka S, Ospina NM, O’Keeffe DT, et al. Subclinical hypothyroidism in pregnancy: a systematic review and meta-analysis. Thyroid 2016; 26: 580-90. 